\title{
Faecal high mobility group box 1 in children with celiac disease: $\mathrm{A}$ pilot study
}

\author{
Francesca Palone ${ }^{a}$, Roberta Vitali ${ }^{c}$, Chiara Maria Trovato ${ }^{a}$, Monica Montuori $^{\mathrm{a}}$, \\ Anna Negroni ${ }^{c}$, Saverio Mallardo ${ }^{a}$, Laura Stronati ${ }^{\mathrm{b}, *}$ \\ a Department of Paediatrics, Sapienza University of Rome, Italy \\ ${ }^{\mathrm{b}}$ Department of Cellular Biotechnology and Haematology, Sapienza University of Rome, Italy \\ ${ }^{c}$ Department of Radiation Biology and Human Health, ENEA, Rome, Italy
}

\section{A R T I C L E I N F O}

\section{Article history:}

Received 20 December 2017

Received in revised form 28 March 2018

Accepted 3 April 2018

Available online $\mathrm{xxx}$

\section{Keywords:}

Celiac disease

HMGB1

Inflammation

Serum anti-transglutaminase

\section{A B S T R A C T}

Background: Celiac disease (CD) is a gluten-related immunological disorder resulting in inflammatory enteropathy.

Aims: We assessed a stool marker of intestinal inflammation, the HMGB1 protein, in children with CD on a gluten free diet (GFD) at baseline and at follow up (FU).

Methods: Thirty-nine children were investigated at diagnosis and at FU. Traditional serum markers of CD (anti-transglutaminase and anti-endomysial antibodies) and faecal HMGB1 (by enzyme-linked immunosorbent assay and immunoblotting) were tested.

Results: There was a marked increase at baseline in both serum anti-transglutaminase IgA (anti-tTGAs) and faecal HMGB1; the latter being undetectable in controls. A strong correlation occurred between the two markers. At 12-month FU in 24 patients on GFD, HMGB1 decreased in all subjects, yet still being detectable in six children: high anti-tTGAs where evident in three, while the three with normal anti-tTGAs were complaining of intestinal symptoms and reported a low GFD adherence.

Conclusions: Faecal HMGB1 is a valuable marker of intestinal inflammation and may have a role in complementing serology in the management of CD children. Future studies including larger patient cohorts and small bowel mucosa histology will be designed to assess the relationship between faecal HMGB1 levels and duodeno-jejunal histopathology.

(c) 2018 Editrice Gastroenterologica Italiana S.r.l. Published by Elsevier Ltd. All rights reserved.

\section{Introduction}

Celiac disease (CD) is an immune-mediated systemic disorder involving the small bowel (SB) mucosa, triggered by peptides from gluten and related prolamines in genetically susceptible subjects which leads to SB inflammation ranging from increased intraepithelial lymphocytes to villous atrophy $[1,2]$.

The diagnostic approach for $\mathrm{CD}$ has recently undergone significant changes since serologic tests have been shown to be useful predictors of SB mucosal damage [3]. Thus, novel paediatric guidelines for $\mathrm{CD}$ have been proposed, defining cases in which a non-invasive approach is recommended or SB mucosa biopsy is needed [4].

\footnotetext{
* Corresponding author at: Department of Cellular Biotechnology and Haematology, Sapienza University of Rome, Viale Regina Elena 324, 00161 Roma, Italy.

E-mail address: laura.stronati@uniroma1.it (L. Stronati).
}

Lifelong adherence to a gluten-free diet (GFD) is the key treatment of $C D$ patients, both to promote mucosal healing and prevent complications; however, assessment of GFD compliances is the cornerstone in patient management. Periodic measurements of serum anti-transglutaminase IgA levels (anti-tTGAs) are widely thought to be useful in monitoring adherence to GFD [5]. However, anti-tTGAs may not always reflect the status of the SB mucosa since various degrees of mucosal damage may persist despite normal serum antitTGAs. The latter may not identify minor voluntary transgressions or unperceived dietary gluten contamination of GFD [6].

The high mobility group box 1 (HMGB1) protein has recently been described as a reliable faecal marker of intestinal inflammation in paediatric and adult inflammatory bowel disease (IBD), either in overt or subclinical status [7,8]. HMGB1 is a protein included in a group of endogenous molecules (also known as alarmins or damage-associated molecular patterns) with intestinal and systemic pro-inflammatory properties when secreted in the extracellular milieu [9]. HMGB1 serum levels have recently been 


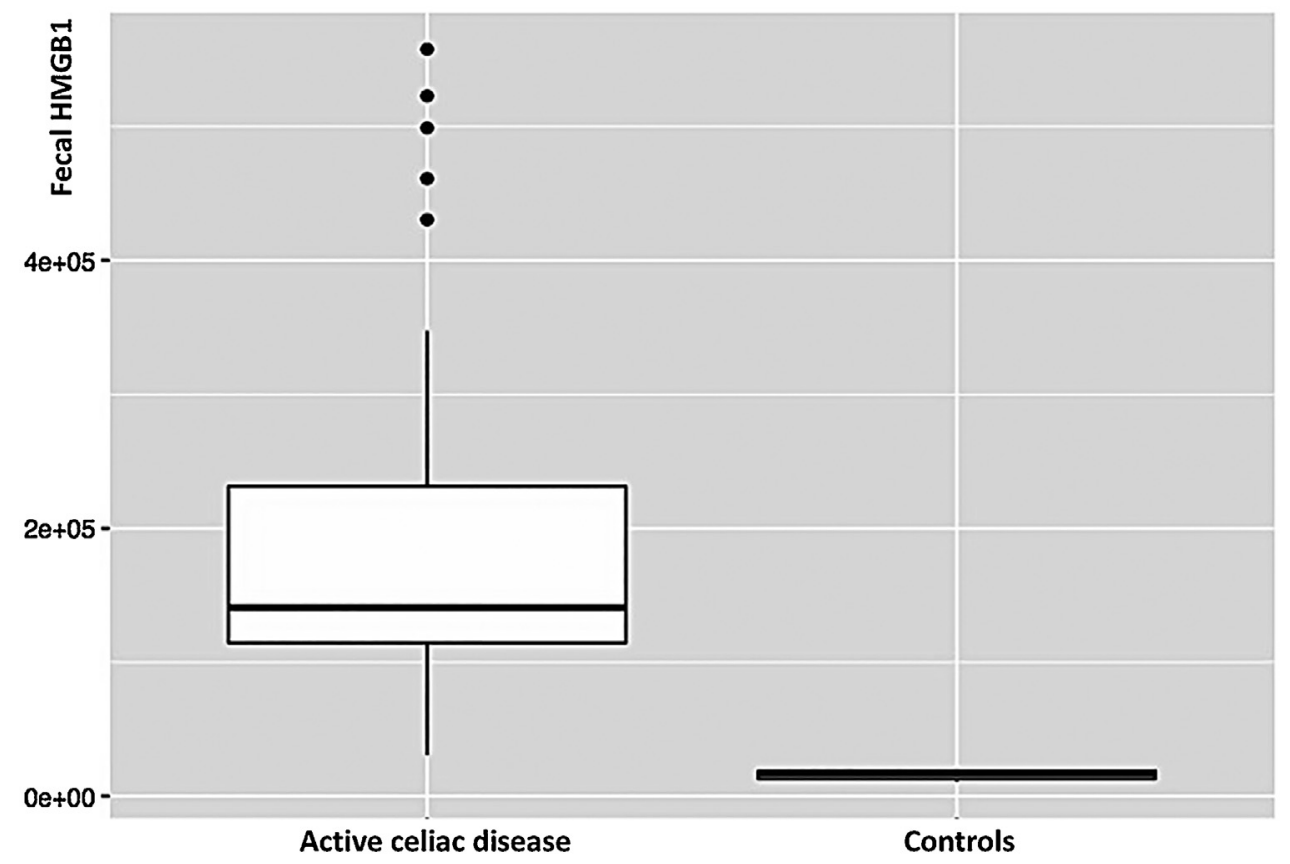

Fig. 1. Faecal HMGB1 level in active coeliac disease at diagnosis and in controls. $P<0.001$ between the two groups (Wilcoxon rank sum test).

shown to have increased in $\mathrm{CD}$ at diagnosis helping to characterize patients according to their phenotypical subtype [10].

In this preliminary report we investigated the usefulness of faecal HMGB1 as a non-invasive marker in the diagnosis and in the follow-up of children with CD.

\section{Material and methods}

We enrolled 39 patients (24 girls; median age: 8.2 years; range: 15 months-18 years) with a $C D$ diagnosis and 20 sexand age-matched controls (median age: 11.0 years; range: 5-18 years) referred to the Paediatric Gastroenterology and Liver Unit of "Sapienza University of Rome" in a 24-month period. All patients were positive for serum anti-tTGAs and anti-endomysial antibodies (EMA); 38 out of 39 underwent upper gastrointestinal (GI) endoscopy and SB mucosa biopsies, in agreement with the ESPGHAN criteria published in 1990 [11]. One child was diagnosed as stated by the novel guidelines released by ESPGHAN in 2012 [4].

At diagnosis, 24 children had a combination of classical gastrointestinal (GI) features such as diarrhoea, abdominal pain, abdominal distension, bloating and growth failure. Fifteen had no GI features and were investigated owing to a family history (in 5) or nonspecific symptoms and signs (in 10) such as pallor, asthenia, poor school performance, mood changes, iron deficiency anaemia, thyroid disease and neurological manifestations.

Stool samples from all children were collected at diagnosis; 24 samples were obtained at 12-month follow-up on GFD. Histology was graded in line with Marsh-Oberhuber criteria [12]. Anti-tTGAs were assayed at diagnosis and at follow-up with enzyme linked immunosorbent assay (ELISA) commercially available kits from Eurospital (Trieste, Italy; cut-off value $>9 \mathrm{UA} / \mathrm{ml}$ ). In outpatient setting GFD compliance was assessed at a 12-month follow-up; a dietary interview by an expert paediatric gastroenterologist was performed, coupled with anti-tTGAs levels.

Protein extraction from stool samples was performed to assess HMGB1 concentration in accordance with a prior reported method [7]. Twenty micrograms of faecal extracts were fractionated by sodium dodecyl sulfate-(12\%) polyacrylamide gel electrophoresis. Proteins were transferred in polyvinylidenefluoride membrane
(Bio-Rad Laboratories) and blocked with TBS-Tween 20 0.1\%, containing 5\% non-fat dry milk. Anti-human HMGB1 antibody (1:1.000; R\&D, Minneapolis, Minnesota) was diluted in TBS-Tween 20 0.1\%, containing 3\% non-fat dry milk and incubated overnight at $4{ }^{\circ} \mathrm{C}$. Membranes were washed in TBS-Tween $200.1 \%$, incubated for $1 \mathrm{~h}$ with horseradish peroxidase-conjugated secondary antibody (Santa Cruz Biotechnology Inc., Santa Cruz, California), washed in TBS-Tween $0.1 \%$, and developed with LiteBlotEXTEND (Euroclone, Milan, Italy). Densitometric analysis of the western blot bands was performed using the Software ImageQuant Las500 (GE Healthcare Life Science, Uppsala, Sweden).

\subsection{Statistical analysis}

Shapiro-Wilk test of normality rejected the normal distribution hypothesis for HMGB1 values. Wilcoxon signed-rank test was therefore used to compare mean HMGB1 values between patients with $C D$ at diagnosis and the control group (significance taken as $\mathrm{P}<0.001)$. The same test was then used to compare mean HMGB1 values at $C D$ diagnosis and during follow-up. The correlation between HMGB1 and anti-tTG value was evaluated by Spearman's rank correlation coefficient (rho) following non-parametric distribution. All statistical analyses were performed using R v 3.3.3 (R Foundation for Statistical Computing, Vienna, Austria).

\section{Results}

Faecal HMGB1 was significantly increased in all 39 CD patients at diagnosis compared to controls, while it was undetectable in the latter (Wilcoxon rank sum test with continuity correction $\mathrm{p}<0.001$ ) (Fig. 1). A strong correlation was found between faecal HMGB1 and serum levels of anti-tTGAs (Spearman's rho =0.79) (Fig. 2).

Patients underwent physical examination and nutritional counselling by an expert paediatric gastroenterologist to investigate probable dietary pitfalls at 6 and 12 months following GFD. Stools were tested at 12 months post-GFD. Twenty-four of 39 patients were available for faecal HMGB1 and serum anti-tTGAs measurements. 


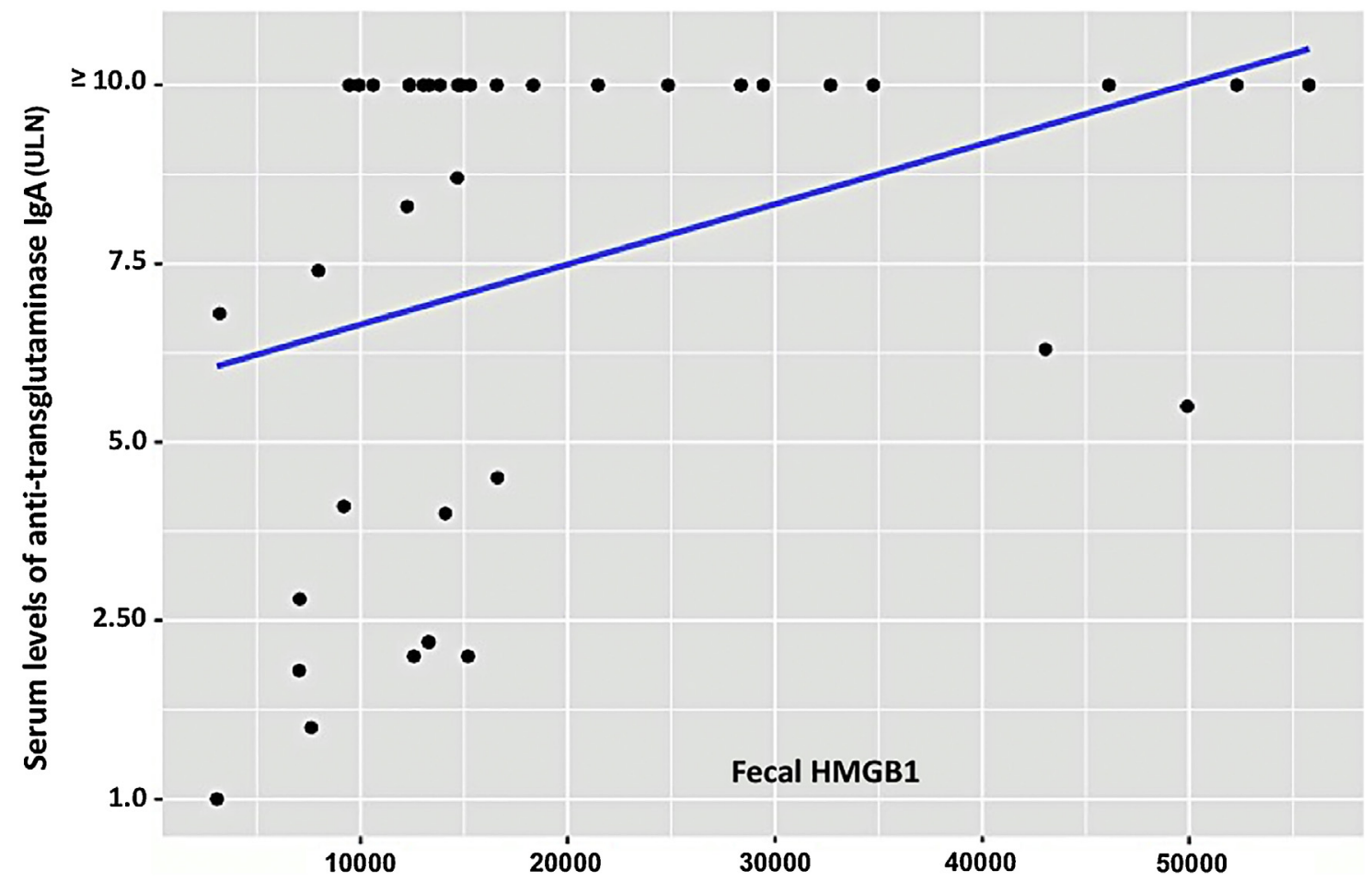

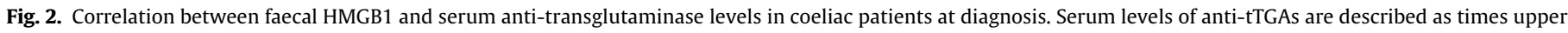
limit of normal (ULN). (Spearman's rank correlation rho $=0.79$ ).

Faecal HMGB1 markedly decreased in all 24 patients (Wilcoxon rank sum test $\mathrm{p}<0.01$ ), although it was still detectable in 6 patients. Of the latter, 3 exhibited abnormal values of serum anti-tTGAs, expressed in multiples of the upper limit of normal (ULN) (a value of serum anti-tTGA 2.5 times ULN in 1, of 2.0 times ULN in 2), while 3 had normal serum anti-tTGAs and reported difficulty in following a strict GFD, claiming voluntary minimal transgressions. Three patients with detectable faecal HMGB1 and normal serum antitTGAs reported GI symptoms such as bloating and epigastric pain (in 2), abdominal pain with faecal constipation (in 1).

\section{Discussion}

Several experimental observations indicate HMGB1 is a proinflammatory protein that is released by activated immunocytes and damaged or necrotic cells, playing a key role in mediating inflammation at both intestinal and multi-organ levels [13]. Moreover, its neutralization has been shown to remarkably reduce the degree of tissue damage and gut inflammation in mouse models [14]. Stool HMGB1 has recently been proposed as a valuable biomarker of mucosal inflammation in IBD, being more sensitive than calprotectin in revealing microscopic tissue injuries [15]. We hypothesized that damage of the intestinal mucosa in $C D$, resulting from the activation of both innate and adaptive immunity, might promote HMGB1 release in the gut lumen and then in the stool content. Thus, we investigated the usefulness of this non-invasive marker in the diagnosis and follow-up of children with CD.

Interestingly, HMGB1 strikingly increased in the stool samples of all patients at diagnosis, however undetectable in controls; moreover, a noticeable positive correlation between faecal HMGB1 and serum anti-tTGAs titers was observed in patients, suggesting that an active enteropathy could be the culprit of the rise in HMGB1.

A key feature of our study is that faecal HMGB1 decreased significantly in $\mathrm{CD}$ patients at the 12-month follow-up on GFD with a similar reduction trend in serum anti-tTGAs. However, six patients still showed detectable faecal HMGB1: three with high serum anti-tTGAs, with the latter being normal in the remaining three. Interestingly, patients with noticeable stool HMGB1 and normal anti-tTGAs were complaining of GI symptoms such as bloating, abdominal pain and constipation, and reported low GFD adherence.

Our observations suggest that persistence of detectable levels of stool HMGB1 in these subjects may hint to an underlying low grade enteropathy, not revealed by anti-tTGAs levels, closely to what has been reported in IBD patients [15]. Intriguingly, the GI symptoms might have been a feature of minor voluntary transgression or involuntary, unperceived dietary gluten ingestion. Indeed, while it is commonly agreed that anti-tTGAs levels increase after continuous and chronic gluten ingestion [16], minimal and fluctuating dietary transgressions might be revealed by stool HMGB1 measurement. Obviously, this speculation needs further well-designed studies, including histological evaluation of SB mucosa as well as assessment of faecal HMGB1 and classical serum markers of CD.

We acknowledge that these preliminary data have some limitations. The lack of duodenal biopsies at follow-up has dampened speculation on a conceivable relationship between SB mucosa status and increased stool HMGB1. Current data restrict the generalization of our findings as a pilot study.

In conclusion, this study confirms that HMGB1 is a valid stool marker of enteropathy. HMGB1 is clearly useful in CD at diagnosis, as well as during follow-up to assess adherence to GFD. If confirmed in larger cohorts prospectively, our data might suggest a new way to assess intestinal inflammation and cell damage in $C D$ through a non-invasive and easily available tool to complement serology.

\section{Conflict of interest}

None declared.

\section{References}

[1] Guandalini S, Assiri A. Celiacdisease: a review. JAMA Pediatr 2014;168:272-8. [2] Villanacci V, Ceppa P, Tavani E, Vindigni C, Volta U, Gruppo Italiano Patologi Apparato Digerente (GIPAD), et al. Coeliac disease: the histology report. Dig Liver Dis 2011;43:385-95. 
[3] Donaldson MR, Firth SD, Wimpee H, Leiferman KM, Zone JJ, Horsley W, et al. Correlation of duodenal histology with tissue transglutaminase and endomysial antibody levels in pediatric celiac disease. Clin Gastroenterol Hepatol 2007:5:567-73.

[4] Husby S, Koletzko S, Korponay-Szabo IR, Mearin ML, Phillips A, Shamir R, et al. European Society for Pediatric Gastroenterology, Hepatology, and Nutrition guidelines for the diagnosis of coeliac disease. J Pediatr Gastroenterol Nutr 2012;54:136-60.

[5] Valitutti F, Trovato CM, Montuori M, Cucchiara S. Pediatric celiac disease: follow-up in the spotlight. Adv Nutr 2017;8:356-61.

[6] Leffler DA, Edwards George JB, Dennis M, Cook EF, Schuppan D, Kelly CP. A prospective comparative study of five measures of gluten-free diet adherence in adults with coeliac disease. Aliment Pharmacol Ther 2007;26:1227-35.

[7] Vitali R, Stronati L, Negroni A, Di Nardo G, Pierdomenico M, del Giudice E, et al. Faecal HMGB1 is a novel marker of intestinal mucosal inflammation in pediatric inflammatory bowel disease. Am J Gastroenterol 2011;106:2029-40.

[8] Palone F, Vitali R, Cucchiara S, Pierdomenico M, Negroni A, Aloi M, et al. Role of HMGB1 as a suitable biomarker of subclinical intestinal inflammation and mucosal healing in patients with inflammatory bowel disease. Inflamm Bowel Dis 2014;20:1448-57.

[9] Magna M, Pisetsky DS. The role of HMGB1 in the pathogenesis of inflammatory and autoimmune diseases. Mol Med 2014;20:138-46.
[10] Manti S, Cuppari C, Tardino L. HMGB1 as a new biomarker of celiac disease in children: a multicenter study. Nutrition 2017;37:18-21.

[11] Walker-Smith JA, Guandalini S, Schmitz J, Schmerling DH, Visakorpi JK. Revised criteria for the diagnosis of celiac disease. Report of a working group. Arch Dis Child 1990;65:909-11.

[12] Oberhuber G. Histopathology of celiac disease. Biomed Pharmacother 2016:200(54):368-72.

[13] Harris HE, Andersson U, Pisetsky DS. HMGB1: a multifunctional alarmin driving autoimmune and inflammatory disease. Nat Rev Rheumatol 2012;8:195-202.

[14] Vitali R, Palone F, Pierdomenico M, Negroni A, Cucchiara S, Aloi M, et al. Dipotas sium glycyrrhizate via HMGB1 or AMPK signaling suppresses oxidative stress during intestinal inflammation. Biochem Pharmacol 2015;97:292-9.

[15] Palone F, Vitali R, Cucchiara S, Mennini M, Armuzzi A, Pugliese D, et al. Faecal HMGB1 reveals microscopic inflammation in adult and pediatric patients with inflammatory bowel disease in clinical and endoscopic remission. Inflamm Bowel Dis 2016;22:2886-93.

[16] Fasano A, Catassi C. Clinical practice. Celiac disease. N Engl J Med 2012;367:2419-26. 